Quantitative Plant Biology

cambridge.org/qpb

\title{
Dynamic cytokinin signalling landscapes during lateral root formation in Arabidopsis
}

\author{
Milica Nenadić ${ }^{1}$ and Joop E. M. Vermeer ${ }^{1,2}(0)$
}

${ }^{1}$ Department of Plant and Microbial Biology \& Zurich-Basel Plant Science Centre, University of Zurich, Zurich,
Switzerland; ${ }^{2}$ Laboratory of Cell and Molecular Biology, University of Neuchâtel, Neuchâtel, Switzerland

Cite this article: M. Nenadic and Joop E. M. Vermeer. Dynamic cytokinin signalling landscapes during lateral root formation in Arabidopsis. Quantitative Plant Biology, 2: 13, 1-9 https://dx.doi.org/10.1017/qpb.2021.13

Received: 19 April 2021

Revised: 4 October 2021

Accepted: 20 October 2021

Keywords:

Arabidopsis; cytokinin; cytokinin transport; lateral roots.

Author for correspondence:

Joop E. M. Vermeer

E-mail: Joop.Vermeer@unine.ch

(c) The Author(s), 2021. Published by Cambridge University Press in association with The John Innes Centre. This is an Open Access article, distributed under the terms of the Creative Commons Attribution-

NonCommercial-NoDerivatives licence (https://creativecommons.org/licenses/ by-nc-nd/4.0/), which permits non-commercial re-use, distribution, and reproduction in any medium, provided the original work is unaltered and is properly cited. The written permission of Cambridge University Press must be obtained for commercial re-use or in order to create a derivative work.
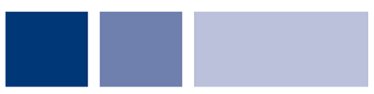

John Innes Centre

Unlocking Nature's Diversity

\section{CAMBRIDGE} UNIVERSITY PRESS

\begin{abstract}
By forming lateral roots, plants expand their root systems to improve anchorage and absorb more water and nutrients from the soil. Each phase of this developmental process in Arabidopsis is tightly regulated by dynamic and continuous signalling of the phytohormones cytokinin and auxin. While the roles of auxin in lateral root organogenesis and spatial accommodation by overlying cell layers have been well studied, insights on the importance of cytokinin is still somewhat limited. Cytokinin is a negative regulator of lateral root formation with versatile modes of action being activated at different root developmental zones. Here, we review the latest progress made towards our understanding of these spatially separated mechanisms of cytokinin-mediated signalling that shape lateral root initiation, outgrowth and emergence and highlight some of the enticing open questions.
\end{abstract}

\section{Introduction}

Most plants rely on their roots to efficiently explore the soil to extract water and nutrients from it. Since this underground environment is both spatially and temporally heterogeneous, plants require a developmentally flexible root system with postembryonic branching capacity. In Arabidopsis, this plasticity is achieved through partially differentiated pericycle cells that can re-enter the cell cycle and give rise to the lateral roots (LRs). These newly formed organs initiate from pre-branching sites that are specified in the basal meristem, where periodic auxin signalling allots conditional meristematic activity to fractions of pericycle cells adjacent to the xylem (Beeckman et al., 2001; De Smet et al., 2007; Moreno-Risueno et al., 2010). As these cells enter the differentiation zone, they might experience yet another auxin maximum and launch the LR developmental programme (De Rybel et al., 2010). Besides the recruitment of the LR founder cells (LRFCs), auxin signalling also enables spatial accommodation of the growing LR by the overlying tissues: endodermis, cortex and epidermis (Cavallari et al., 2021; Du \& Scheres, 2018).

LR primordium (LRP) development is classified into eight stages based on anatomical characteristics and cell divisions (Malamy \& Benfey, 1997). The first stage occurs when a single or two LRFCs undergo anticlinal divisions to create two short daughter cells surrounded by two larger cells (Stage I) (Torres-Martinez et al., 2019; Torres-Martinez et al., 2020). Ensuing anticlinal divisions, cell expansion and periclinal divisions give rise to a four-layered LRP (Stage IV), which is the final stage before the organ grows through the endodermis. After this resistant cell layer has been crossed, more divisions and cellular expansion result in the formation of a complex stage VI primordium that resembles the primary root meristem in its organization. Once the LR breaches the surface of the primary root, it is considered emerged.

Although it is difficult to predict which xylem pole pericycle (XPP) cells along the root will become LRFCs, positioning and distribution of LR stages are not without order. LRFC specification is restricted to the early differentiation zone, also known as the developmental window. Once the LRFCs are recruited to the LR developmental programme, adjacent or opposite XPP cells no longer initiate additional LRs. These restrictions result in the acropetal architecture of the root system, where spaced LRPs are located at the alternating xylem poles (Dubrovsky et al., 2006). Cytokinin (CK) signalling is one of the several circuits implicated in regulating both aspects of LR positioning (Bielach et al., 2012). 
Major natural CKs in plants are the two $\mathrm{N}^{6}$-prenylated adenine derivatives called trans-zeatin ( $\mathrm{tZ}$ ) and isopentenyl adenine (iP) (Osugi \& Sakakibara, 2015). The first and ratelimiting step in their biosynthesis is catalysed by one of the seven ADENOSINE PHOSPHATE-ISOPENTENYLTRANSFERASES (IPTs) (Kakimoto, 2001; Sakakibara, 2006; Takei et al., 2001). The resulting iP ribotide may then be hydroxylated by CYTOCHROME P450 CYP735A1 or CYP735A2 and turned into a $\mathrm{tZ}$ ribotide (Takei et al., 2004a). In order to become biologically active, both iP- and tZ-riboside 5/-monophosphates have to be converted to the corresponding nucleobases by the activity of LONELY GUY (LOG) enzymes that comprise a family with nine members in Arabidopsis (Kuroha et al., 2009). Once synthesised, CK bases might bind to the membrane-bound receptors (ARABIDOPSIS HISTIDINE KINASE2 [AHK2], AHK3, and CYTOKININ RESPONSE1 [CRE1]/AHK4), which trigger CK signalling (Inoue et al., 2001; Nishimura et al., 2004). The signal is then transduced by five ARABIDOPSIS HISTIDINE PHOSPHOTRANSFER PROTEINS (AHPs) to the nucleus, where 11 type-B ARABIDOPSIS RESPONSE REGULATORS (type-B ARRs) mediate CK-regulated gene expression (Hutchison et al., 2006; Mason et al., 2005; Miyata et al., 1998). Some of the upregulated genes are type-A ARRs, which encode negative feedback elements in CK signalling (D’Agostino et al., 2000; To et al., 2007). Another negative regulator of CK signalling is AHP6, a member of the AHP family that lacks the canonical phospho-accepting histidine residue (Mahonen et al., 2006). Finally, CK breakdown is catalysed by seven CYTOKININ OXIDASES (CKXs) (Schmulling et al., 2003; Werner et al., 2006). Please note that there are additional CK species and enzymes involved in their metabolism, which have been excluded from this brief introduction, and have been extensively discussed elsewhere (Kieber \& Schaller, 2014; Nedved et al., 2021; Vylicilova et al., 2020).

$\mathrm{CK}$ acts as a negative regulator of LR formation, with versatile modes of action. Besides inhibiting primary root growth, CK treatment also inhibits LR initiation and arrests development of LRs at Stages I-IV, but does not impede the growth of stages V onward (Bielach et al., 2012; Chang et al., 2013; Laplaze et al., 2007; Li et al., 2006). Similarly, an increase in endogenous CKs through ectopic expression of an IPT from Agrobacterium tumefaciens (Agrobacterium) can result in plants with no LRs developed past Stage IV or V (Bielach et al., 2012; Kuderova et al., 2008; Laplaze et al., 2007). As expected, plants with decreased CK levels have longer roots and increased LR density, with ectopic primordia initiating often near already developing LRs (Chang et al., 2013; 2015; Werner et al., 2003). It is clear that this hormone affects LR formation at different zones along the primary root. Thus here, we will focus on these spatially separated mechanisms of CK-mediated signalling that shape LR development. We point the reader to several great reviews that discuss the role of auxin signalling and the auxin and CK interplay in LR development and emergence (Cavallari et al., 2021; Du \& Scheres, 2018; Jing \& Strader, 2019; Stoeckle et al., 2018).

\section{LRP positioning is affected by CK}

Densities of LRPs, emerged LRs and total LRs are frequently quantified traits that help characterise the root system architecture. Inhibition of LR formation by exogenous CK treatments was shown to be dose-dependent (Chang et al., 2013). Ubiquitous ectopic expression of genes involved in CK biosynthesis and metabolism, such as IPT, LOG and CKX, have independently confirmed this aspect of CK action (Kuderova et al., 2008; Kuroha et al., 2009; Werner et al., 2003).

More targeted manipulation of endogenous CK levels with the use of GAL4-GFP enhancer trap lines, which can drive the expression of target genes in defined plant tissues, provided further insights into where this spatial control occurred. By employing J0121 GAL4-GFP enhancer trap line, Laplaze et al. (2007) observed decreased LRP density in plants expressing an Agrobacterium IPT in XPP cells. These results were later partially confirmed, with the phenotypic inconsistencies attributed to the different growth conditions used in the two studies. Specifically, Bielach et al. (2012) first detected GFP signal in XPP cells that had already left the basal meristem of the same J0121 line, which likely explained the lack of an LRP density phenotype due to delayed expression of the Agrobacterium IPT gene. In addition, they reported the clear expression of GFP in LRPs of the J0121 line (Bielach et al., 2012). This shows that care should be taken when using such driver lines (or conventional promoter fragments), as expression patterns may be considerably affected by growth conditions. Based on IPT expression in other GAL4-GFP enhancer trap lines, Bielach et al. (2012) found that regardless of the targeted tissue, strong IPT induction in the basal meristem most efficiently decreased LRP density. Such responsiveness to CK signalling early in the root was not surprising, as root tips had been proposed to integrate information about soil quality and accordingly adjust the frequency of LR initiation events in order to optimise the uptake of water and nutrients (Xuan et al., 2016). It is yet to be demonstrated whether the resulting iP ribotides from different tissues in the basal meristem act in a non-cell autonomous way or become immediately converted to the active CK bases that then travel and trigger cellular responses in XPP cells.

Auxin efflux carrier PIN-FORMED 7 (PIN7) is a potential downstream regulator of $\mathrm{CK}$ effects since its expression was reported to be upregulated in response to exogenous CK treatment and pin 7 mutants displayed an increased density of LRP initiation compared to wild type plants (Benkova et al., 2003; Bishopp et al., 2011; Marhavy et al., 2013; Ruzicka et al., 2009; Simaskova et al., 2015). PIN7 expression was detected in the central root cap, stele and LRP initials, and the frequency of FC specification was perturbed in pin7 plants (Marhavy et al., 2013; Simaskova et al., 2015). CYTOKININ RESPONSE FACTORS (CRFs), which mediate transcriptional control downstream of CK, regulate PINs via a specific PIN CYTOKININ RESPONSE ELEMENT (PCRE) domain. Removal of the PCRE from the PIN7 promoter reduced the sensitivity of LR initiation and development to exogenous CK (Simaskova et al., 2015). In contrast to these observations, another study found that $\mathrm{CK}$ treatment significantly reduces the expression of PIN7 in the vascular tissue through induction of SHY2 via the AHK3/ARR1 two-component signalling pathway (Dello Ioio et al., 2008). Additional studies will be required to better understand these opposite results.

\section{CK regulates $L R$ initiation and spacing}

Although mutants affected in CK biosynthesis may not display a strongly increased LRP density compared to wild type, they often exhibit more closely spaced LRPs and recruit more LRFCs upon auxin treatment, suggesting that $\mathrm{CK}$ also affects LR formation in the differentiation zone (Bielach et al., 2012; Chang et al., 2015). Using the fluorescent CK-signalling reporter TCS::GFP (Muller \& Sheen, 


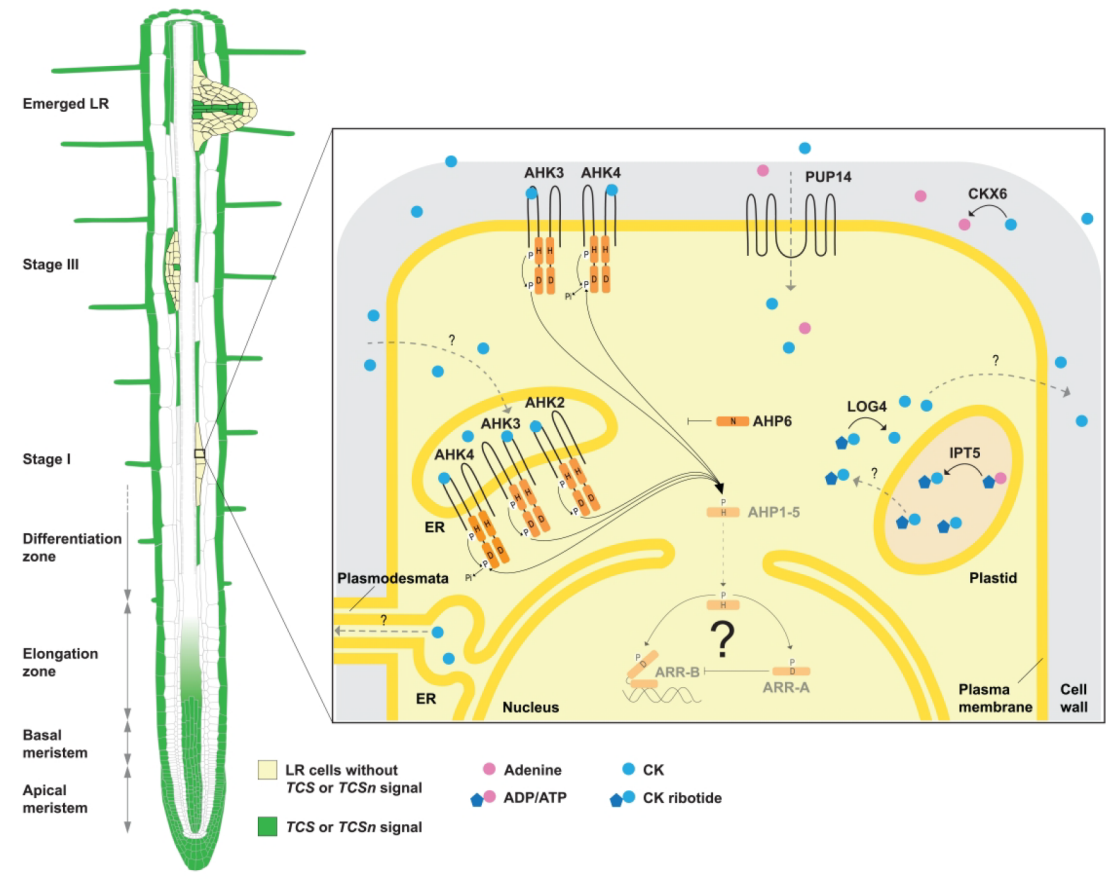

Fig. 1. Schematic illustration of the expression of cytokinin (CK)-signalling reporters in primary root and an overview of CK metabolism, transport and signalling in a cell of Stage I lateral root primordium (LRP). Fluorescent CK-signalling reporters TCS::GFP and TCSn::ntd Tomato were used to monitor CK responses in primary root (Bielach et al., 2012; Marhavy et al., 2014; Montesinos et al., 2020). Here, we focus on a stage I LRP to illustrate a scenario where a cell that expresses CK receptors and biosynthesis genes might suppress $\mathrm{CK}$ signalling and serve as a potential source of the hormone to neighbouring pericycle cells. Plasmodesmata represent one of the potential ways through which CK might reach the neighbouring cells, although more research is needed to confirm such mode of CK transport. ARABIDOPSIS HISTIDINE KINASES (AHKs) are depicted at both the endoplasmatic reticulum (ER) and plasma membranes, although the latter localization has yet to be shown in the LRP cells. In the case of CK binding, AHKs can be autophosphorylated at a conserved His residue $(\mathrm{H})$ and this phosphate is then carried over to a conserved Asp (D). Exact downstream pathways that could mediate transcriptional dependent and -independent effects of CK signalling in stage I LRPs are unknown. Arrows with a solid line indicate chemical reaction, arrows with a dashed line indicate movement. Additional abbreviations: LR, lateral root; AHP, ARABIDOPSIS HISTIDINE PHOSPHOTRANSFER PROTEIN; ARR-A/B, type-A/B ARABIDOPSIS RESPONSE REGULATOR; PUP, PURINE PERMEASE; CKX, CYTOKININ OXIDASE; IPT, ADENOSINE PHOSPHATE-ISOPENTENYLTRANSFERASE; LOG, LONELY GUY; ADP, adenOsine diphosphate; ATP, adenosine triphosphate. The drawing of the primary root is a modified version from Peret (2017), while the illustration of CK signalling circuitry is modified from on a published schematic (Hwang et al., 2012).

2008) to monitor CK responses in cells and tissues involved in LR organogenesis, no fluorescence signal was observed in the root zone corresponding to the developmental window, encompassing LRFCs and Stages I-III. The expression of TCS::GFP was enhanced in XPP cells in between already initiated LRPs (Figure 1). This response was correlated with LR formation, since TCS expression could only be observed in the few pericycle cells adjacent to an already initiated LRP (Bielach et al., 2012; Marhavy et al., 2014). It has been hypothesised that the CK molecules perceived by these cells originated both from themselves and the neighbouring LRP, and that this CK signalling prevented them from entering the LR developmental programme, that is, $\mathrm{CK}$ could be a positional cue regulating LR spacing (Bielach et al., 2012; Chang et al., 2015). We would also like to point out that an improved version of the $\mathrm{CK}$ reporter, TCS new (TCSn), was developed, which was reported to be more sensitive to phosphorelay signalling in Arabidopsis. Plant expressing TCSn::GFP showed stronger GFP fluorescence in the root meristem and vasculature compared to TCS::GFP, whereas their expression patterns were qualitatively very similar during the LR development (Zürcher et al., 2013). However, a study comparing the two CK reporters in the LR developmental window has not been reported.

In untreated plants, LR initiation is restricted to a relatively narrow developmental window, implying that there is a regulatory mechanism to control such patterning. These unknown regulatory mechanisms operate within the zone distal to the youngest LRP and determine the competence of XPP cells to launch the LR developmental programme (Dubrovsky et al., 2006). When wild type plants were treated with 1-naphthaleneacetic acid (NAA; $1 \mu \mathrm{M})$ for 25 hours, LRP initiation was induced between already recruited LRFCs and stage I LRPs, that is, additional initiation events were limited mainly to the early differentiation zone. Interestingly, in the ipt3,ipt5,ipt7 triple mutant, the same auxin treatment induced LRP initiation along the whole root. Similar results were observed in the arr1,arr11 double mutant, which lacks two B-type ARRs that mediate the signal transfer downstream of the CK receptors and were expressed in the pericycle and elsewhere in roots. In this experiment, Bielach et al. (2012) also found that in the arr1,arr11 double mutant, $11.6 \%$ of LRPs were misplaced (two LRPs separated by one pericycle cell) compared to $1.8 \%$ in wild type. Thus, they proposed that CK activity in pericycle cells might be important to prevent LR initiation outside the early differentiation zone and in close proximity to existing LRPs. In agreement with this hypothesis, Chang et al. (2013) reported that LRP and LR density showed an increase in response to auxin treatment in the ahk2,ahk3 double mutant compared to wild type plants.

The same authors published a model predicting that the suppression of LR initiation in XPP cells neighbouring existing LRFCs was a combined effect of local CK synthesis and by the neighbouring cells (Chang et al., 2015). Of the genes involved in CK biosynthesis, IPT5 and LOG4 were shown to be expressed in most cells of LRPs, from Stage I until emergence (Figure 1) (Chang 
et al., 2015). Therefore, LRPs themselves could be a source of iP nucleobases. Besides LRPs and XPP cells, LOG4 was also expressed in the xylem and xylem pole endodermal cells in the root tip (De Rybel et al., 2014). $\log 4$ mutants displayed more abnormally positioned LRPs, separated by zero, one or two XPP cells, compared to wild-type roots. LRP spacing was not significantly altered in ipt5 mutants, probably due to a higher degree of functional redundancy in this gene family. In addition to LRPs, IPT5 was also shown to be expressed in the pericycle (Chang et al., 2015). Other rootexpressed members of this gene family are IPT3, expressed in the phloem and pericycle, and IPT7, which was expressed both in the endodermis in the elongation zone and in the phloem (Miyawaki et al., 2004; Takei et al., 2004b). ipt3,ipt5 double mutants had almost as many abnormally positioned LRPs as observed in $\log 4$ mutants, and this phenotype was further enhanced in roots of ipt3,ipt5,ipt7 mutants. Interestingly, LR spacing phenotype was also observed in a cyp735a2 mutant. CYP735A2 is expressed in the phloem and pericycle, but not in LRPs, and converts the products of IPT enzymes into the $\mathrm{tZ}$ ribotides (Chang et al., 2015; Takei et al., 2004a).

Considering that these CK biosynthesis genes have an expression pattern that is not restricted to LRPs or the immediately surrounding XPP cells, it can only be speculated about the mechanisms that prevent LR initiation in cells surrounding newly recruited LRFCs. Moreover, the observed abnormal LR positioning phenotypes in $\log 4$, ipt3, ipt5,ipt7 and cyp735a2 mutants were very subtle and had a low penetrance, that is, the majority of LRPs did not have abnormal positions compared to wild-type roots. Thus, although important knowledge has been gathered, additional experiments are required to decipher the molecular mechanism underlying CKmediated LR spacing. Using the DR5pro::LUCIFERASE system, it would be interesting to see whether the altered spacing is already visible when pre-branching sites are specified, or if this is caused by a signalling event that occurs later in the root. Other pathways controlling LRP spacing were recently reviewed by Cavallari et al. (2021).

Besides restricting the ability of XPP cells to launch the LR developmental programme, CK signalling in XPP cells might also be required for proper secondary growth establishment. In Arabidopsis roots, secondary development is organised by two meristems: vascular cambium and phellogen/cork cambium (Ragni \& Greb, 2018). The first originates from procambium and the XPP, and the latter from the entire pericycle, although the first formative divisions occur in XPP cells and then extend to the rest of the pericycle (Smetana et al., 2019; Wunderling et al., 2018). In the ipt3,ipt5,ipt7 triple mutant, which showed LR formation along the whole root upon auxin treatment, secondary root growth was abolished and could be recovered by addition of $\mathrm{tZ}$, in a dosedependent manner (Bielach et al., 2012; Matsumoto-Kitano et al., 2008). Smetana et al. (2019) proposed that a mobile xylem derived signal induced stem-cell identity in the neighbouring cells that give rise to the vascular cambium and that CKs could be these mobile stem-cell-promoting factors. Establishment of the vascular cambium is a prerequisite for XPP cells to be able to start dividing and contributing to radial thickening of the root. It was suggested that a non-cell-autonomous signal triggered by the cambium could render XPP competent to become a secondary meristem (Xiao et al., 2020). It could be speculated whether by limiting the developmental window in which XPP cells could be recruited to form LRPs, CKs also enable XPP cells to transition into a new role as secondary meristems.

\section{Multiple roles of CK in the LRP outgrowth}

The pace of LR development can vary even in two successively initiated primordia. LR initiation, meristem establishment and LR emergence represent growth-control points that can be regulated independently, which provides an additional level of plasticity during the root system development (Dubrovsky et al., 2006). Exogenous CK treatment inhibited LR initiation and arrested growth of LRs at Stages I-IV, while an increase in endogenous CKs resulted in plants with no LRs developed past Stage IV or V (Bielach et al., 2012; Chang et al., 2013; Kuderova et al., 2008; Laplaze et al., 2007; Li et al., 2006).

Although LRPs could produce active iP nucleobases and express the CK receptors $A H K 2, A H K 3$ and $C R E 1 / A H K 4$ already at Stage I, the $C K$ response in this developing organ was reported to be activated only from Stage III and onwards, based on the absence of TSC::GFP signal (Figure 1) (Marhavy et al., 2014). The endoplasmic reticulum (ER) membrane is the principal CK perception site, but a recent study showed that in meristematic cells, pools of ERlocated AHK4 could enter the secretory pathway and reach the plasma membrane (Kubiasova et al., 2020). In parallel, experiments on protoplasts isolated from Arabidopsis roots confirmed CK perception by plasma membrane localised AHK3 and AHK4. Moreover, the CK free bases were detected in apoplastic fractions from roots (Antoniadi et al., 2020). It was hypothesised that the plasma membrane localised receptors might activate distinct branches of downstream signalling compared to the CK receptors localised in the ER, which would enable control of specific processes in meristematic cells (Kubiasova et al., 2020). Thus, it is probable that the suppression of CK signalling in young LRPs is a result of both intracellular AHP6 and extracellular CKX6 (Figure 1) (Andersen et al., 2018; Chang et al., 2013; Moreira et al., 2013; Werner et al., 2003). Even though LRP density and distribution of different stages were not significantly altered in ahp6 mutants compared to wild type, there were defects in the orientation of cell divisions in early stages of LR development. It was proposed that AHP6, through repression of CK signalling, enabled a correct localization of auxin efflux carrier PIN1 and thus the formation of the auxin gradient that is required for LRP patterning (Moreira et al., 2013). While the suppression of CK signalling was needed in the early stages of LR development, from Stage III onward, proper establishment of PIN1 polarity required an intact CK perception. It was shown that independently of transcription, CK enhanced endocytic recycling of PIN1 at specific polar domains, thereby directing the auxin flux towards the tip of LRPs and promoting their development. This CKmediated control of auxin distribution was evident in plants treated with exogenous CKs, which caused rapid and excessive depletion of plasma membrane-localised PIN1, leading to an arrest of LR development (Marhavy et al., 2011; Marhavy et al., 2014). Based on the reported specific expression of CKX6 in Stage I LRPs, it would be interesting to investigate the contribution of this gene to the LR development and spacing (Chang et al., 2015).

TRANSPORTER OF IBA1 (TOB1) was recently identified as a tonoplast-localized transporter of the auxin precursor indole3-butyric acid (IBA) and was shown to limit LR formation. CK treatment resulted in the expansion of TOB1 expression from LRPflanking regions to the whole primordium, suggesting an additional pathway of CK-mediated regulation of LRP organogenesis (Michniewicz et al., 2019). The cell cycle could also be a potential target of CK action in LRP outgrowth, since a high concentration of exogenous CK caused an arrest of LRFCs at the G2/M phase 
(Li et al., 2006). Transcription of genes specific for the G2/M phase was reported to be mediated by two R1R2R3-MYB transcriptional activators, MYB3R1 and MYB3R4. The corresponding myb3r1,myb3r4 double mutant has fewer cells in the shoot and root apical meristems (SAM and RAM). However, their expression is not limited to just these two tissues, for example, both genes are also expressed in the pericycle (Haga et al., 2007). It has been recently shown that CK directly promotes nuclear trafficking of MYB3R4 to activate mitotic cell cycle-related gene expression in the SAM. Interestingly, while CK treatment increased the number of cells in the epidermal (L1) layer of wild-type SAMs, the same CK treatment led to a premature SAM termination or reduction of cells in the L1 layer in the myb3r1,myb3r4 double mutant compared to the control (Yang et al., 2021). It would be interesting to see if these genes are expressed in LRPs and whether MYB3R4 could also modulate CK signalling responses during LR development.

In contrast to the exogenous CK treatments, manipulation of endogenous CK levels suggested that, unlike XPP cells, young LRPs are not as sensitive to this hormone (Laplaze et al., 2007). Increased CK levels in the early stages of LRP development caused LR growth defects in some GAL4-GFP enhancer trap lines, while they were absent in others (Bielach et al., 2012; Laplaze et al., 2007). Since they used an Agrobacterium IPT that can catalyse the first step in CK synthesis, the subsequent activity of LOG enzymes would still be required to synthesise active nucleobases. Therefore, LOG4 levels in LRP might have acted as a bottle-neck of CK synthesis in these experiments, possibly masking developmental defects. Additionally, cell cycle arrest and PIN1 depletion upon exogenous CK treatment were absent in crel/ahk4 mutants (Li et al., 2006; Marhavy et al., 2011). Considering that a portion of CRE1/AHK4 receptors has been shown to reside on the plasma membrane of meristematic cells and might activate distinct branches of signalling downstream CK perception compared to those localised to the ER, it is possible that young LRPs were insensitive to endogenously increased CK levels because the produced CKs remained inside the cells. Our knowledge about the molecular mechanisms involved in CK transport is still incomplete, although potential efflux and influx transporters have been identified (Duran-Medina et al., 2017; Liu et al., 2019; Nedved et al., 2021).

The only characterised CK efflux carrier facilitating the transport of CK across the plasma membrane is ATP-BINDING CASSETTE G14 (ABCG14), which is expressed primarily in the pericycle and stele of roots, but it was not revealed whether $A B G C 14$ is also expressed in LRPs. Depending on the study, abcg14 mutant seedlings had either shorter or longer primary roots compared to wild type, without differences in LRP densities being reported (Ko et al., 2014; Zhang et al., 2014). Other genes implicated in CK transport are PURINE PERMEASES (PUPS), AZA-GUANINE RESISTANT (AZG) family and EQUILIBRATIVE NUCLEOSIDE TRANSPORTERS (ENTs) (Burkle et al., 2003; Gillissen et al., 2000; Hirose et al., 2008; Tessi et al., 2021a; 2021b; Zürcher et al., 2016). Besides free CKs, members of the first two families could transport other purines, while ENTs were able to transport nucleosides as well as CK ribosides. Of the three characterised PUPs, which all demonstrated direct CK uptake activity in different assays, only PUP14 was reported to be expressed in LRPs, localizing to the plasma membrane (Figure 1) (Burkle et al., 2003; Zürcher et al., 2016). Inducible knockdown of PUP14 expression resulted in shorter roots and suppressed LR formation (Zürcher et al., 2016). It would be important to use the CRISPR-Cas9 technology to generate pup14 knockout mutants and check whether these plants show similar phenotypes. Likewise, it would be interesting to elucidate the expression patterns of other members of the PUP family in the context of LR formation and to reveal their subcellular localization, as it was recently reported that two rice PUPs localise to the ER (Xiao et al., 2019; Xiao et al., 2020). The AZG family has only two members in Arabidopsis, AZG1 expressed in vasculature of already emerged LRs, and $A Z G 2$ expressed in a few cortical and epidermal cells overlying LRPs (Tessi et al., 2021a; 2021b). AZG2 was proposed to function as a CK diffusion facilitator functioning in both transport directions across ER and plasma membranes. However, it was not shown to which compartment AGZ2 predominantly localises in the cells overlying LRPs, as only transcriptional reporters were used to study the expression during LR emergence. azg2 roots had a slightly increased number of Stages VI to VIII LRP compared to control plants, but this difference was not significant (Tessi et al., 2021a). With regard to the ENT family, ENT3 and ENT6 could participate in CK riboside transport, with both genes being expressed in the root vasculature (Hirose et al., 2008; Korobova et al., 2021). Finally, naturally occurring CKs possess relatively hydrophobic side-chains and their diffusion from the cytoplasm to the apoplast cannot be ruled out (Nedved et al., 2021).

Considering our incomplete knowledge on CK transport and different branches of CK signalling in young LRPs, discrepancies between experiments employing exogenous CK treatments and those manipulating endogenous CK levels cannot be easily resolved. Another proposed explanation concerns the fact that while the affinity of AHKs to their ligands is around 1-40 nM, relatively high CK concentrations (more than $50 \mathrm{nM}$ 6-benzylaminopurine) were required to cause a strong cell cycle inhibition and altered cellular patterning in LRPs (Chang et al., 2013; Lomin et al., 2015). Moreover, the concentrations of iP and $\mathrm{tZ}$ in root extracts were in the range from 0.4 to $2.6 \mathrm{pmol} / \mathrm{g}$ fresh weight (Svacinova et al., 2012). Thus, Chang et al. (2013) suggested that the experiments employing high CK treatments might not be informative about the physiologically relevant CK effects. However, local CK concentration in LRPs could be higher than those measurements in root extracts, as CKs are unevenly distributed in organs at either the tissue or cellular level. More targeted measurements of CK concentration in LRPs, like what was achieved for different cell types in the root apex, could advance this discussion, assuming that isolation of single cells from differentiated tissues is feasible (Antoniadi et al., 2015).

\section{CK signalling in the overlying endodermis}

LR emergence starts with the first formative cell divisions leading to a Stage I LRP, as the newly formed organ needs to overcome the mechanical constraints imposed by the overlying cell layers. In order to make way for the emerging LRP, neighbouring endodermal cells lose volume and/or alter their shape and relinquish their tight junction-like diffusion barrier called the Casparian strip. These accommodating responses are crucial for LR development and are regulated by AUX/IAA SHORT HYPOCOTYL 2 (SHY2)mediated auxin signalling in differentiated endodermal cells (Vermeer et al., 2014). It has been proposed that auxin coordinates reorganization of the cytoskeleton in the pericycle and endodermis, however, important knowledge on the remodelling of endodermis is still missing (Vilches Barro et al., 2019). Interestingly, the CK signalling reporter, TCS::GFP, was shown to be also induced in the endodermal cells overlying the early-stage LRPs (Figure 1) (Bielach et al., 2012). This suggests that the same cells that display SHY2-mediated auxin signalling, also experience CK 
signalling. In the transition zone of the primary root, it was reported that CK could antagonise auxin by inducing the expression of SHY2 (Dello Ioio et al., 2008). However, it is unknown if similar auxin-cytokinin interactions exist in the endodermis during LR emergence. Recently, it was shown that CK affects the dynamics of the microtubule cytoskeleton and counteracts auxin-driven microtubule rearrangements in epidermal cells of the primary root (Montesinos et al., 2020). If cortical microtubules contribute to the remodelling of endodermis, it would be interesting to see whether and how auxin and CK affect their organization and dynamics. For example, does CK signalling in the endodermis affect spatial accommodating responses by stabilising the microtubule cytoskeleton, or does it have a completely different role? The endodermal cells overlying LRPs also undergo dynamic suberisation. First, suberin is deposited, then it is removed and replaced by another polymer, cutin (Berhin et al., 2019; Ursache et al., 2021). However, unlike in the rest of the endodermis, suberisation around LR emergence sites was reported to be independent of CK signalling (Andersen et al., 2018).

\section{CK regulates angular growth of emerged LRs}

The angle at which LRs emerge from the primary root is another root architectural trait that affects plant performance and was recently found to be regulated by CK. Upon emergence at a $90^{\circ}$ angle, LRs start perceiving gravity and other environmental cues, like hypoxia, to establish the gravitropic set point angle (GSA). CK signalling at the upper flank of emerged LRs integrates these environmental signals and interferes with cellular elongation and proliferation through transcriptional regulators, such as CRF2 and CRF3. Hence, CK presents an anti-gravitropic component in emerged LRs that controls the radial expansion of the root system (Waidmann et al., 2019). Interestingly, cold stress, which is known to decrease LR numbers and GSA, was also shown to induce the expression of CRF2 and CRF3 in primary roots, LRPs and emerged LRs. The cold signal for expressing CRF2 was mediated by a subset of the TCS system (AHK2, AHK3, AHPs, ARR1, ARR10 and ARR12), while CRF3 was upregulated via TCS-independent pathways. Both transcription factors were necessary for LR initiation in plants exposed to low temperatures, whereas their involvement in establishing GSA under cold stress were not reported (Jeon et al., 2016).

\section{Concluding remarks}

Auxin is a master regulator of LR organogenesis that can integrate a variety of environmental signals, thus coordinating the endogenous plant developmental programme with exogenous inputs from the environment (Cavallari et al., 2021). Nonetheless, it is clear that auxin is not the only crucial regulator, with CK affecting every step of LR development. To date, major progress has been made in our understanding of the CK-mediated control of developmental processes. Still, some questions remain open. Further insights could arise from the advances in imaging that allow quantification of shapes, volume and changes in the (an)isotropy of growing cells. To better understand CK transport during plant development, a similar biosensor to the recently described AuxSens that enables direct quantification of auxin in living cells would be a major breakthrough (Herud-Sikimic et al., 2021). Since there is a strong interaction between $\mathrm{CK}$ and auxin signalling and responses, it is important to monitor their levels simultaneously when possible. It is likely that cellular decisions require a certain CK to auxin ratio.
In addition, the use of cell type-specific promoters to specifically image or manipulate particular tissues will be instrumental to better understand how hormone signalling affects organ development. Likewise, these promoters are essential for the CRISPR system that generates tissue-specific knockouts, CRISPR-TSKO. This approach has already been proven efficient in creating somatic mutations in LRFCs resulting in LR-specific knock-outs of genes that also have roles in other developmental processes. Moreover, by linking it to promoters that are active a defined stages of LR development, or inducible expression systems, TSKO could be used to target genes at specific stages of LR development (Decaestecker et al., 2019). Uncovering new regulators of LR development could also be prompted by the high-throughput single-cell RNA sequencing methods of the root, although it could be a challenge to isolate single cells from differentiated tissues (Denyer et al., 2019; Wang et al., 2020; Wendrich et al., 2020).

\section{Acknowledgements}

The authors thank Ari Pekka Mähönen for stimulating discussions about the CK signalling in plants and its potential roles during the secondary root growth.

Financial support. This work was supported by the grants from the Swiss National Science Foundation (Schweizerischer Nationalfonds zur Förderung der Wissenschaftlichen Forschung; PP00P3_157524 and 310030_197568) and a PSC-Syngenta Research Fellowship.

Conflict of interest. The authors declare no conflict of interest.

Authorship contributions. M.N. prepared the first draft of the manuscript and figure. J.E.M.V. provided input to the draft and both authors contributed to writing the final version.

Data availability statement. No new data or code are presented in this paper.

\section{References}

Andersen, T. G., Naseer, S., Ursache, R., Wybouw, B., Smet, W., De Rybel, B., Vermeer, J. E. M., \& Geldner, N. (2018). Diffusible repression of cytokinin signalling produces endodermal symmetry and passage cells. Nature, 555, 529-533.

Antoniadi, I., Novak, O., Gelova, Z., Johnson, A., Plihal, O., Simersky, R., Mik, V., Vain, T., Mateo-Bonmati, E., Karady, M., Pernisova, M., Plackova, L., Opassathian, K., Hejatko, J., Robert, S., Friml, J., Dolezal, K., Ljung, K., \& Turnbull, C. (2020). Cell-surface receptors enable perception of extracellular cytokinins. Nature Communications, 11, 4284.

Antoniadi, I., Plackova, L., Simonovik, B., Dolezal, K., Turnbull, C., Ljung, K., \& Novak, O. (2015). Cell-type-specific cytokinin distribution within the Arabidopsis primary root apex. The Plant Cell, 27, 1955-1967.

Beeckman, T., Burssens, S., \& Inze, D. (2001). The peri-cell-cycle in Arabidopsis. Journal of Experimental Botany, 52, 403-411.

Benkova, E., Michniewicz, M., Sauer, M., Teichmann, T., Seifertova, D., Jurgens, G., \& Friml, J. (2003). Local, efflux-dependent auxin gradients as a common module for plant organ formation. Cell, 115, 591-602.

Berhin, A., de Bellis, D., Franke, R. B., Buono, R. A., Nowack, M. K., \& Nawrath, C. (2019). The root cap cuticle: A cell wall structure for seedling establishment and lateral root formation. Cell, 176, 1367-1378, e1368.

Bielach, A., Podlesakova, K., Marhavy, P., Duclercq, J., Cuesta, C., Muller, B., Grunewald, W., Tarkowski, P., \& Benkova, E. (2012). Spatiotemporal regulation of lateral root organogenesis in Arabidopsis by cytokinin. The Plant Cell, 24, 3967-3981.

Bishopp, A., Help, H., El-Showk, S., Weijers, D., Scheres, B., Friml, J., Benkova, E., Mahonen, A. P., \& Helariutta, Y. (2011). A mutually inhibitory interaction between auxin and cytokinin specifies vascular pattern in roots. Current Biology, 21, 917-926. 
Burkle, L., Cedzich, A., Dopke, C., Stransky, H., Okumoto, S., Gillissen, B., Kuhn, C., \& Frommer, W. B. (2003). Transport of cytokinins mediated by purine transporters of the PUP family expressed in phloem, hydathodes, and pollen of Arabidopsis. The Plant Journal, 34, 13-26.

Cavallari, N., Artner, C., \& Benkova, E. (2021). Auxin-regulated lateral root organogenesis. Cold Spring Harbor Perspectives in Biology, 13, a039941.

Chang, L., Ramireddy, E., \& Schmulling, T. (2013). Lateral root formation and growth of Arabidopsis is redundantly regulated by cytokinin metabolism and signalling genes. Journal of Experimental Botany, 64, 5021-5032.

Chang, L., Ramireddy, E., \& Schmulling, T. (2015). Cytokinin as a positional cue regulating lateral root spacing in Arabidopsis. Journal of Experimental Botany 66, 4759-4768.

D'Agostino, I. B., Deruere, J., \& Kieber, J. J. (2000). Characterization of the response of the Arabidopsis response regulator gene family to cytokinin. Plant Physiology, 124, 1706-1717.

De Rybel, B., Adibi, M., Breda, A. S., Wendrich, J. R., Smit, M. E., Novak, O., Yamaguchi, N., Yoshida, S., Van Isterdael, G., Palovaara, J., Nijsse, B., Boekschoten, M. V., Hooiveld, G., Beeckman, T., Wagner, D., Ljung, K. Fleck, C., \& Weijers, D. (2014). Plant development. Integration of growth and patterning during vascular tissue formation in Arabidopsis. Science, 345 1255215.

De Rybel, B., Vassileva, V., Parizot, B., Demeulenaere, M., Grunewald, W., Audenaert, D., Van Campenhout, J., Overvoorde, P., Jansen, L., Vanneste, S., Moller, B., Wilson, M., Holman, T., Van Isterdael, G., Brunoud, G. Vuylsteke, M., Vernoux, T., De Veylder, L., Inze, D., Weijers, D., . . Beeckman, T. (2010). A novel aux/IAA28 signaling cascade activates GATA23dependent specification of lateral root founder cell identity. Current Biology, 20, 1697-1706.

De Smet, I., Tetsumura, T., De Rybel, B., Frei dit Frey, N., Laplaze, L., Casimiro, I., Swarup, R., Naudts, M., Vanneste, S., Audenaert, D., Inze, D., Bennett, M. J., \& Beeckman, T. (2007). Auxin-dependent regulation of lateral root positioning in the basal meristem of Arabidopsis. Development, 134, 681-690.

Decaestecker, W., Buono, R. A., Pfeiffer, M. L., Vangheluwe, N., Jourquin, J., Karimi, M., Van Isterdael, G., Beeckman, T., Nowack, M. K., \& Jacobs, T. B. (2019). CRISPR-TSKO: A technique for efficient mutagenesis in specific cell types, tissues, or organs in Arabidopsis. The Plant Cell, 31, 2868-2887.

Dello Ioio, R., Nakamura, K., Moubayidin, L., Perilli, S., Taniguchi, M., Morita, M. T., Aoyama, T., Costantino, P., \& Sabatini, S. (2008). A genetic framework for the control of cell division and differentiation in the root meristem. Science, 322, 1380-1384.

Denyer, T., Ma, X., Klesen, S., Scacchi, E., Nieselt, K., \& Timmermans, M. C. P. (2019). Spatiotemporal developmental trajectories in the arabidopsis root revealed using high-throughput single-cell RNA sequencing. Developmental Cell, 48, 840-852, e845.

Du, Y., \& Scheres, B. (2018). Lateral root formation and the multiple roles of auxin. Journal of Experimental Botany, 69, 155-167.

Dubrovsky, J. G., Gambetta, G. A., Hernandez-Barrera, A., Shishkova, S., \& Gonzalez, I. (2006). Lateral root initiation in Arabidopsis: Developmental window, spatial patterning, density and predictability. Annals of Botany, 97, 903-915.

Duran-Medina, Y., Diaz-Ramirez, D., \& Marsch-Martinez, N. (2017) Cytokinins on the move. Frontiers in Plant Science, 8, 146.

Gillissen, B., Burkle, L., Andre, B., Kuhn, C., Rentsch, D., Brandl, B., \& Frommer, W. B. (2000). A new family of high-affinity transporters for adenine, cytosine, and purine derivatives in Arabidopsis. The Plant Cell, 12, 291-300.

Haga, N., Kato, K., Murase, M., Araki, S., Kubo, M., Demura, T., Suzuki, K., Muller, I., Voss, U., Jurgens, G., \& Ito, M. (2007). R1R2R3-Myb proteins positively regulate cytokinesis through activation of KNOLLE transcription in Arabidopsis thaliana. Development, 134, 1101-1110.

Herud-Sikimic, O., Stiel, A. C., Kolb, M., Shanmugaratnam, S., Berendzen, K. W., Feldhaus, C., Hocker, B., \& Jurgens, G. (2021). A biosensor for the direct visualization of auxin. Nature, 592, 768-772.

Hirose, N., Takei, K., Kuroha, T., Kamada-Nobusada, T., Hayashi, H., \& Sakakibara, H. (2008). Regulation of cytokinin biosynthesis, compartmentalization and translocation. Journal of Experimental Botany, 59, 75-83.
Hutchison, C. E., Li, J., Argueso, C., Gonzalez, M., Lee, E., Lewis, M. W., Maxwell, B. B., Perdue, T. D., Schaller, G. E., Alonso, J. M., Ecker, J. R., \& Kieber, J. J. (2006). The Arabidopsis histidine phosphotransfer proteins are redundant positive regulators of cytokinin signaling. The Plant Cell, 18, 3073-3087.

Hwang, I., Sheen, J., \& Muller, B. (2012). Cytokinin signaling networks. Annual Review of Plant Biology, 63, 353-380.

Inoue, T., Higuchi, M., Hashimoto, Y., Seki, M., Kobayashi, M., Kato, T., Tabata, S., Shinozaki, K., \& Kakimoto, T. (2001). Identification of CRE1 as a cytokinin receptor from Arabidopsis. Nature, 409, 1060-1063.

Jeon, J., Cho, C., Lee, M. R., Van Binh, N., \& Kim, J. (2016). CYTOKININ RESPONSE FACTOR2 (CRF2) and CRF3 regulate lateral root development in response to cold stress in Arabidopsis. The Plant Cell, 28, 1828-1843.

Jing, H., \& Strader, L. C. (2019). Interplay of auxin and cytokinin in lateral root development. International Journal of Molecular Sciences, 20, 486.

Kakimoto, T. (2001). Identification of plant cytokinin biosynthetic enzymes as dimethylallyl diphosphate: ATP/ADP isopentenyltransferases. Plant and Cell Physiology, 42, 677-685.

Kieber, J. J., \& Schaller, G. E. (2014). Cytokinins. The Arabidopsis Book/American Society of Plant Biologists, 12, e0168.

Ko, D., Kang, J., Kiba, T., Park, J., Kojima, M., Do, J., Kim, K. Y., Kwon, M., Endler, A., Song, W. Y., Martinoia, E., Sakakibara, H., \& Lee, Y. (2014). Arabidopsis ABCG14 is essential for the root-to-shoot translocation of cytokinin. Proceedings of the National Academy of Sciences of the United States of America, 111, 7150-7155.

Korobova, A., Kuluev, B., Mohlmann, T., Veselov, D., \& Kudoyarova, G. (2021). Limitation of cytokinin export to the shoots by nucleoside transporter ENT3 and its linkage with root elongation in Arabidopsis. Cells, 10, 350 .

Kubiasova, K., Montesinos, J. C., Samajova, O., Nisler, J., Mik, V., Semeradova, H., Plihalova, L., Novak, O., Marhavy, P., Cavallari, N., Zalabak, D., Berka, K., Dolezal, K., Galuszka, P., Samaj, J., Strnad, M., Benkova, E., Plihal, O., \& Spichal, L. (2020). Cytokinin fluoroprobe reveals multiple sites of cytokinin perception at plasma membrane and endoplasmic reticulum. Nature Communications, 11, 4285.

Kuderova, A., Urbankova, I., Valkova, M., Malbeck, J., Brzobohaty, B., Nemethova, D., \& Hejatko, J. (2008). Effects of conditional IPT-dependent cytokinin overproduction on root architecture of Arabidopsis seedlings. Plant and Cell Physiology, 49, 570-582.

Kuroha, T., Tokunaga, H., Kojima, M., Ueda, N., Ishida, T., Nagawa, S., Fukuda, H., Sugimoto, K., \& Sakakibara, H. (2009). Functional analyses of LONELY GUY cytokinin-activating enzymes reveal the importance of the direct activation pathway in Arabidopsis. The Plant Cell, 21, 3152-3169.

Laplaze, L., Benkova, E., Casimiro, I., Maes, L., Vanneste, S., Swarup, R., Weijers, D., Calvo, V., Parizot, B., Herrera-Rodriguez, M. B., Offringa, R., Graham, N., Doumas, P., Friml, J., Bogusz, D., Beeckman, T., \& Bennett, M. (2007). Cytokinins act directly on lateral root founder cells to inhibit root initiation. The Plant cell, 19, 3889-3900.

Li, X., Mo, X., Shou, H., \& Wu, P. (2006). Cytokinin-mediated cell cycling arrest of pericycle founder cells in lateral root initiation of Arabidopsis. Plant and Cell Physiology, 47, 1112-1123.

Liu, C. J., Zhao, Y., \& Zhang, K. (2019). Cytokinin transporters: Multisite players in cytokinin homeostasis and signal distribution. Frontiers in Plant Science, 10, 693.

Lomin, S. N., Krivosheev, D. M., Steklov, M. Y., Arkhipov, D. V., Osolodkin, D. I., Schmulling, T., \& Romanov, G. A. (2015). Plant membrane assays with cytokinin receptors underpin the unique role of free cytokinin bases as biologically active ligands. Journal of Experimental Botany, 66, 1851-1863.

Mahonen, A. P., Bishopp, A., Higuchi, M., Nieminen, K. M., Kinoshita, K., Tormakangas, K., Ikeda, Y., Oka, A., Kakimoto, T., \& Helariutta, Y. (2006). Cytokinin signaling and its inhibitor AHP6 regulate cell fate during vascular development. Science, 311, 94-98.

Malamy, J. E., \& Benfey, P. N. (1997). Organization and cell differentiation in lateral roots of Arabidopsis thaliana. Development, 124, 33-44.

Marhavy, P., Bielach, A., Abas, L., Abuzeineh, A., Duclercq, J., Tanaka, H., Parezova, M., Petrasek, J., Friml, J., Kleine-Vehn, J., \& Benkova, E. (2011). Cytokinin modulates endocytic trafficking of PIN1 auxin efflux carrier to control plant organogenesis. Developmental Cell, 21, 796-804. 
Marhavy, P., Duclercq, J., Weller, B., Feraru, E., Bielach, A., Offringa, R., Friml, J., Schwechheimer, C., Murphy, A., \& Benkova, E. (2014). Cytokinin controls polarity of PIN1-dependent auxin transport during lateral root organogenesis. Current Biology, 24, 1031-1037.

Marhavy, P., Vanstraelen, M., De Rybel, B., Zhaojun, D., Bennett, M. J., Beeckman, T., \& Benkova, E. (2013). Auxin reflux between the endodermis and pericycle promotes lateral root initiation. The EMBO Journal, 32, 149-158.

Mason, M. G., Mathews, D. E., Argyros, D. A., Maxwell, B. B., Kieber, J. J., Alonso, J. M., Ecker, J. R., \& Schaller, G. E. (2005). Multiple type-B response regulators mediate cytokinin signal transduction in Arabidopsis. The Plant Cell, 17, 3007-3018.

Matsumoto-Kitano, M., Kusumoto, T., Tarkowski, P., Kinoshita-Tsujimura, K., Vaclavikova, K., Miyawaki, K., \& Kakimoto, T. (2008). Cytokinins are central regulators of cambial activity. Proceedings of the National Academy of Sciences of the United States of America, 105, 20027-20031.

Michniewicz, M., Ho, C. H., Enders, T. A., Floro, E., Damodaran, S., Gunther, L. K., Powers, S. K., Frick, E. M., Topp, C. N., Frommer, W. B., \& Strader, L. C. (2019). TRANSPORTER OF IBA1 links auxin and cytokinin to influence root architecture. Developmental Cell, 50, 599-609, e594.

Miyata, S., Urao, T., Yamaguchi-Shinozaki, K., \& Shinozaki, K. (1998). Characterization of genes for two-component phosphorelay mediators with a single HPt domain in Arabidopsis thaliana. FEBS Letters, 437, 11-14.

Miyawaki, K., Matsumoto-Kitano, M., \& Kakimoto, T. (2004). Expression of cytokinin biosynthetic isopentenyltransferase genes in Arabidopsis: Tissue specificity and regulation by auxin, cytokinin, and nitrate. The Plant Journal, 37, 128-138.

Montesinos, J. C., Abuzeineh, A., Kopf, A., Juanes-Garcia, A., Otvos, K., Petrasek, J., Sixt, M., \& Benkova, E. (2020). Phytohormone cytokinin guides microtubule dynamics during cell progression from proliferative to differentiated stage. The EMBO Journal, 39, e104238.

Moreira, S., Bishopp, A., Carvalho, H., \& Campilho, A. (2013). AHP6 inhibits cytokinin signaling to regulate the orientation of pericycle cell division during lateral root initiation. PLOS One, 8, e56370.

Moreno-Risueno, M. A., Van Norman, J. M., Moreno, A., Zhang, J., Ahnert, S. E., \& Benfey, P. N. (2010). Oscillating gene expression determines competence for periodic Arabidopsis root branching. Science, 329, 1306-1311.

Muller, B., \& Sheen, J. (2008). Cytokinin and auxin interaction in root stemcell specification during early embryogenesis. Nature, 453, 1094-1097.

Nedved, D., Hosek, P., Klima, P., \& Hoyerova, K. (2021). Differential subcellular distribution of cytokinins: How does membrane transport fit into the big picture?. International Journal of Molecular Sciences, 22, 3428.

Nishimura, C., Ohashi, Y., Sato, S., Kato, T., Tabata, S., \& Ueguchi, C. (2004). Histidine kinase homologs that act as cytokinin receptors possess overlapping functions in the regulation of shoot and root growth in Arabidopsis. The Plant Cell, 16, 1365-1377.

Osugi, A., \& Sakakibara, H. (2015). Q\&A: How do plants respond to cytokinins and what is their importance? BMC Biology, 13, 1-10.

Peret, B. (2017). Primary and lateral root.ai. Figshare.

Ragni, L., \& Greb, T. (2018). Secondary growth as a determinant of plant shape and form. Seminars in Cell \& Developmental Biology, 79, 58-67.

Ruzicka, K., Simaskova, M., Duclercq, J., Petrasek, J., Zazimalova, E., Simon, S., Friml, J., Van Montagu, M. C., \& Benkova, E. (2009). Cytokinin regulates root meristem activity via modulation of the polar auxin transport. Proceedings of the National Academy of Sciences of the United States of America, 106, 4284-4289.

Sakakibara, H. (2006). Cytokinins: Activity, biosynthesis, and translocation. Annual Review of Plant Biology, 57, 431-449.

Schmulling, T., Werner, T., Riefler, M., Krupkova, E., \& Bartrina y Manns, I. (2003). Structure and function of cytokinin oxidase/dehydrogenase genes of maize, rice, Arabidopsis and other species. Journal of Plant Research, 116, 241-252.

Simaskova, M., O’Brien, J. A., Khan, M., Van Noorden, G., Otvos, K., Vieten, A., De Clercq, I., Van Haperen, J. M., Cuesta, C., Hoyerova, K., Vanneste, S., Marhavy, P., Wabnik, K., Van Breusegem, F., Nowack, M., Murphy, A., Friml, J., Weijers, D., Beeckman, T., \& Benkova, E. (2015). Cytokinin response factors regulate PIN-FORMED auxin transporters. Nature Communications, 6, 8717.
Smetana, O., Makila, R., Lyu, M., Amiryousefi, A., Sanchez Rodriguez, F., Wu, M. F., Sole-Gil, A., Leal Gavarron, M., Siligato, R., Miyashima, S., Roszak, P., Blomster, T., Reed, J. W., Broholm, S., \& Mahonen, A. P. (2019). High levels of auxin signalling define the stem-cell organizer of the vascular cambium. Nature, 565, 485-489.

Stoeckle, D., Thellmann, M., \& Vermeer, J. E. (2018). Breakout-lateral root emergence in Arabidopsis thaliana. Current Opinion in Plant Biology, 41, $67-72$.

Svacinova, J., Novak, O., Plackova, L., Lenobel, R., Holik, J., Strnad, M., \& Dolezal, K. (2012). A new approach for cytokinin isolation from Arabidopsis tissues using miniaturized purification: Pipette tip solid-phase extraction. Plant Methods, 8, 17.

Takei, K., Sakakibara, H., \& Sugiyama, T. (2001). Identification of genes encoding adenylate isopentenyltransferase, a cytokinin biosynthesis enzyme, in Arabidopsis thaliana. Journal of Biological Chemistry, 276, 26405-26410.

Takei, K., Ueda, N., Aoki, K., Kuromori, T., Hirayama, T., Shinozaki, K., Yamaya, T., \& Sakakibara, H. (2004b). AtIPT3 is a key determinant of nitrate-dependent cytokinin biosynthesis in Arabidopsis. Plant and Cell Physiology, 45, 1053-1062.

Takei, K., Yamaya, T., \& Sakakibara, H. (2004a). Arabidopsis CYP735A1 and CYP735A2 encode cytokinin hydroxylases that catalyze the biosynthesis of trans-Zeatin. The Journal of Biological Chemistry, 279, 41866-41872.

Tessi, T. M., Brumm, S., Winklbauer, E., Schumacher, B., Pettinari, G., Lescano, I., Gonzalez, C. A., Wanke, D., Maurino, V. G., Harter, K., \& Desimone, M. (2021a). Arabidopsis AZG2 transports cytokinins in vivo and regulates lateral root emergence. New Phytologist, 229, 979-993.

Tessi, T. M., Shahriari, M., Maurino, V. G., Meissner, E., Novak, O., Pasternak, T., Schumacher, B. S., Flubacher, N. S., Nautscher, M., Williams, A., Kazimierczak, Z., Strnad, M., Thumfart, J. O., Palme, K., Desimone, M., \& Teale, W. D. (2021b). The auxin transporter PIN1 and the cytokinin transporter AZG1 interact to regulate the root stress response. bioRxiv, https://doi.org/10.1101/2020.10.22.350363.

To, J. P., Deruere, J., Maxwell, B. B., Morris, V. F., Hutchison, C. E., Ferreira, F. J., Schaller, G. E., \& Kieber, J. J. (2007). Cytokinin regulates type-A Arabidopsis response regulator activity and protein stability via two-component phosphorelay. The Plant Cell, 19, 3901-3914.

Torres-Martinez, H. H., Hernandez-Herrera, P., Corkidi, G., \& Dubrovsky, J. G. (2020). From one cell to many: Morphogenetic field of lateral root founder cells in Arabidopsis thaliana is built by gradual recruitment. Proceedings of the National Academy of Sciences of the United States of America, 117, 20943-20949.

Torres-Martinez, H. H., Rodriguez-Alonso, G., Shishkova, S., \& Dubrovsky, J. G. (2019). Lateral root primordium morphogenesis in angiosperms. Frontiers in Plant Science, 10, 206.

Ursache, R., De Jesus Vieira Teixeira, C., Denervaud Tendon, V., Gully, K., De Bellis, D., Schmid-Siegert, E., Grube Andersen, T., Shekhar, V., Calderon, S., Pradervand, S., Nawrath, C., Geldner, N., \& Vermeer, J. E. M. (2021). GDSL-domain proteins have key roles in suberin polymerization and degradation. Nature Plants, 7, 353-364.

Vermeer, J. E., von Wangenheim, D., Barberon, M., Lee, Y., Stelzer, E. H., Maizel, A., \& Geldner, N. (2014). A spatial accommodation by neighboring cells is required for organ initiation in Arabidopsis. Science, 343, 178-183.

Vilches Barro, A., Stockle, D., Thellmann, M., Ruiz-Duarte, P., Bald, L., Louveaux, M., von Born, P., Denninger, P., Goh, T., Fukaki, H., Vermeer, J. E. M., \& Maizel, A. (2019). Cytoskeleton dynamics are necessary for early events of lateral root initiation in Arabidopsis. Current Biology, 29, $2443-$ 2454, e2445.

Vylicilova, H., Bryksova, M., Matuskova, V., Dolezal, K., Plihalova, L., \& Strnad, M. (2020). Naturally occurring and artificial N9-cytokinin conjugates: From synthesis to biological activity and back. Biomolecules, 10, 832.

Waidmann, S., Ruiz Rosquete, M., Scholler, M., Sarkel, E., Lindner, H., LaRue, T., Petrik, I., Dunser, K., Martopawiro, S., Sasidharan, R., Novak, O., Wabnik, K., Dinneny, J. R., \& Kleine-Vehn, J. (2019). Cytokinin functions as an asymmetric and anti-gravitropic signal in lateral roots. Nature Communications, 10, 3540.

Wang, X., Ye, L., Lyu, M., Ursache, R., Loytynoja, A., \& Mahonen, A. P. (2020). An inducible genome editing system for plants. Nature Plants 6, 766772 . 
Wendrich, J. R., Yang, B., Vandamme, N., Verstaen, K., Smet, W., Van de Velde, C., Minne, M., Wybouw, B., Mor, E., Arents, H. E., Nolf, J., Van Duyse, J., Van Isterdael, G., Maere, S., Saeys, Y., \& De Rybel, B. (2020). Vascular transcription factors guide plant epidermal responses to limiting phosphate conditions. Science, 370, eaay 4970.

Werner, T., Kollmer, I., Bartrina, I., Holst, K., \& Schmulling, T. (2006). New insights into the biology of cytokinin degradation. Plant Biology, 8, 371-381.

Werner, T., Motyka, V., Laucou, V., Smets, R., Van Onckelen, H., \& Schmülling, T. (2003). Cytokinin-deficient transgenic arabidopsis plants show multiple developmental alterations indicating opposite functions of cytokinins in the regulation of shoot and root meristem activity. The Plant Cell, 15, 2532-2550.

Wunderling, A., Ripper, D., Barra-Jimenez, A., Mahn, S., Sajak, K., Targem, M. B., \& Ragni, L. (2018). A molecular framework to study periderm formation in Arabidopsis. The New phytologist, 219, 216-229.

Xiao, W., Molina, D., Wunderling, A., Ripper, D., Vermeer, J. E. M., \& Ragni, L. (2020). Pluripotent pericycle cells trigger different growth outputs by integrating developmental cues into distinct regulatory modules. Current Biology, 30, 4384-4398, e4385.

Xiao, Y., Liu, D., Zhang, G., Gao, S., Liu, L., Xu, F., Che, R., Wang, Y., Tong, H., \& Chu, C. (2019). Big Grain3, encoding a purine permease, regulates grain size via modulating cytokinin transport in rice. Journal of Integrative Plant Biology, 61, 581-597.

Xuan, W., Band, L. R., Kumpf, R. P., Van Damme, D., Parizot, B., De Rop, G., Opdenacker, D., Moller, B. K., Skorzinski, N., Njo, M. F., De Rybel, B., Audenaert, D., Nowack, M. K., Vanneste, S., \& Beeckman, T. (2016). Cyclic programmed cell death stimulates hormone signaling and root development in Arabidopsis. Science, 351, 384-387.

Yang, W., Cortijo, S., Korsbo, N., Roszak, P., Schiessl, K., Gurzadyan, A., Wightman, R., Jonsson, H., \& Meyerowitz, E. (2021). Molecular mechanism of cytokinin-activated cell division in Arabidopsis. Science, 371, 13501355.

Zhang, K., Novak, O., Wei, Z., Gou, M., Zhang, X., Yu, Y., Yang, H., Cai, Y., Strnad, M., \& Liu, C. J. (2014). Arabidopsis ABCG14 protein controls the acropetal translocation of root-synthesized cytokinins. Nature Communications, 5, 3274.

Zürcher, E., Liu, J., di Donato, M., Geisler, M., \& Muller, B. (2016). Plant development regulated by cytokinin sinks. Science, 353, 1027-1030.

Zürcher, E., Tavor-Deslex, D., Lituiev, D., Enkerli, K., Tarr, P. T., \& Muller, B. (2013). A robust and sensitive synthetic sensor to monitor the transcriptional output of the cytokinin signaling network in planta. Plant Physiology, 161, 1066-1075. 